\title{
Linear Variable Differential Transformer Temperature Compensation Technique
}

\author{
Wandee Petchmaneelumka, ${ }^{*}$ Pitsini Mano, and Vanchai Riewruja \\ Department of Instrumentation and Control Engineering, Faculty of Engineering, \\ King Mongkut's Institute of Technology Ladkrabang, Bangkok 10520, Thailand
}

(Received November 27, 2017; accepted March 12, 2018)

Keywords: LVDT, temperature compensation, inductive transducer, analog circuit design

A feedback technique to compensate for the temperature effect on the output signal of the linear variable differential transformer (LVDT) without losing the sensitivity is presented in this paper. The proposed technique is based on the use of a voltage-controlled amplifier to scale the amplitude of the excitation signal for temperature compensation. The proposed feedback technique provides the proportional-plus-integral control action to minimize the error caused by the temperature variation. The proportional-plus-integral action is realized using the integral scheme in the proposed technique. The peak amplitude of the LVDT output signal is sampled by the sample-and-hold circuit (SHC) to obtain the feedback and displacement signals, where the control signal of the SHC is provided by the LVDT output signal. The proposed LVDT temperature compensation technique is emphasized in terms of simple configuration and low cost. Note that the proposed technique is suitable for signal conditioners embedded in smart sensors and smart materials. The performance of the proposed technique is confirmed by experimental implementation using commercially available devices. The maximum error of the core displacement signal can be reduced from $6.52 \%$ for the uncompensated scheme to $0.098 \%$ for the compensated scheme at the ambient temperature of $70{ }^{\circ} \mathrm{C}$.

\section{Introduction}

The linear variable differential transformer (LVDT) is an inductive transducer that is widely applied in instrumentation and measurement systems. The LVDT provides the behavior in terms of high resolution, high linearity, and durability, and is employed for measuring the displacement. The applications of the LVDT can be found in the fields of industries, military, vehicles, structural health monitoring, and scientific and medical equipment for the measurement of position, level, force, flow, and pressure. ${ }^{(1-6)}$ The LVDT consists of a primary winding and two secondary windings connected in series with opposite directions similar to a transformer. The moving core of the LVDT is used for sensing the displacement. The output signal of the LVDT is subtracted between the two secondary windings in the form of amplitude modulation with suppressed carrier (AMSC). Previously, the phase-sensitive 
demodulator formed by a diode and a low-pass filter was used to demodulate the displacement signal from the LVDT signal. The disadvantage of this method is that the large error due to the threshold voltage of the diode occurs. ${ }^{(1)}$ To overcome this error, the analog multiplier and low-pass filter formed as the synchronous demodulator can demodulate the displacement signal without a diode in the signal path. The use of a low-pass filter in traditional approaches deteriorates the response time and stability of the demodulator. Recently, the use of a sampleand-hold circuit (SHC) instead of the analog multiplier and low-pass filter in the synchronous demodulator is more attracted. ${ }^{(7-12)}$ The SHC provides the transfer characteristic in terms of the 'sinc' function, the behavior of which is close to an ideal low-pass filter. ${ }^{(13)}$ Thus, the use of the SHC in the synchronous demodulator can remove the high-frequency components of the LVDT signal to achieve the displacement signal without disturbing the performance of the demodulator. The reference frequency used for the synchronous demodulators mentioned above is provided by the excitation signal. Practically, the phase shift between the excitation signal and the LVDT signal occurs owing to the structure of the LVDT. This phase shift causes the error in the demodulated signal. In addition, the output signal of the LVDT can also be interfered by the variation in ambient temperature. The aforementioned approaches are achieved only with the demodulator without temperature compensation. Therefore, the applications of the LVDT for a high-temperature environment are avoided. There are two recent approaches using dual secondary windings in the LVDT structure for the compensation of the temperature effect. ${ }^{(14,15)}$ Unfortunately, both approaches require a specific design of the LVDT structure; such approaches are unsuitable for commercial purposes. Alternately, the approach based on the ratiometric technique has been proposed. ${ }^{(16)}$ This approach requires the analog multiplier, the analog divider, and the synchronous demodulator, which deteriorates the response time and system stability. In addition, the aforementioned approaches are unsuitable for embedded systems and smart sensors used in a smart factory owing to the complicated topology of the signal conditioner. In this paper, the proposed feedback technique is introduced for the minimization of the temperature effect. The feedback signal is provided by the sum of two secondary winding signals, which obtains a constant amplitude signal. The proposed feedback scheme provides a proportional-plus-integral control action to reduce the error due to the ambient temperature variation. Moreover, the error caused by the phase shift is prevented because the reference frequency is directly achieved from the secondary winding signals. The proposed scheme is attractive in terms of simple configuration and low cost.

\section{Circuit Description}

The simplified diagram of the LVDT is depicted in the dash-line block of Fig. 1(a). The LVDT output signal is obtained from the difference between two secondary winding signals. If the signal $v_{e x}=V_{P} \sin \omega_{e x} t$ is applied to the primary winding, where $\omega_{e x}=2 \pi f_{e x}$ and $f_{e x}$ is the excitation frequency, then the two secondary winding signals $v_{S 1}$ and $v_{S 2}$ can be written as

$$
v_{S 1}=K_{L} l_{C}\left(1+\frac{l}{l_{C}}\right)(1-\alpha \Delta T) V_{P} \sin \left(\omega_{e x} t+\phi_{S}\right),
$$




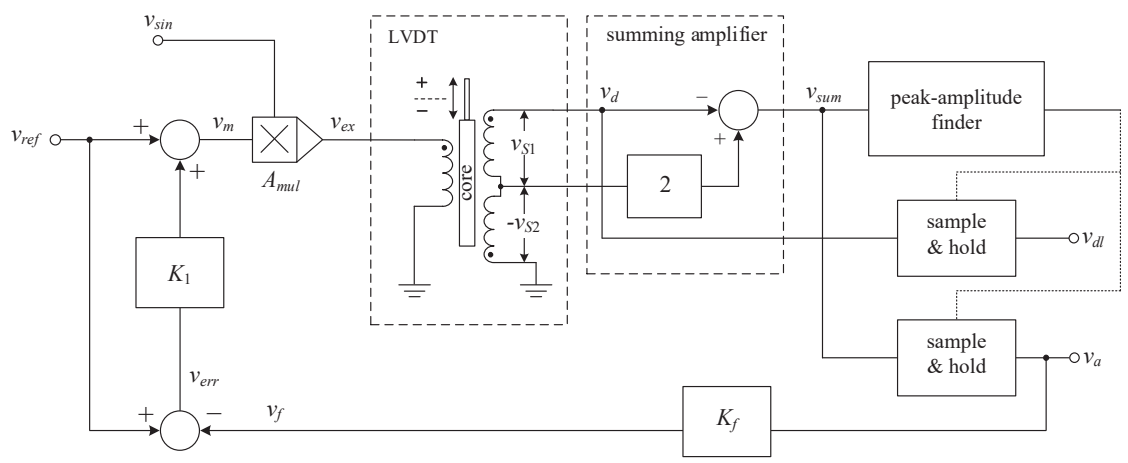

(a)

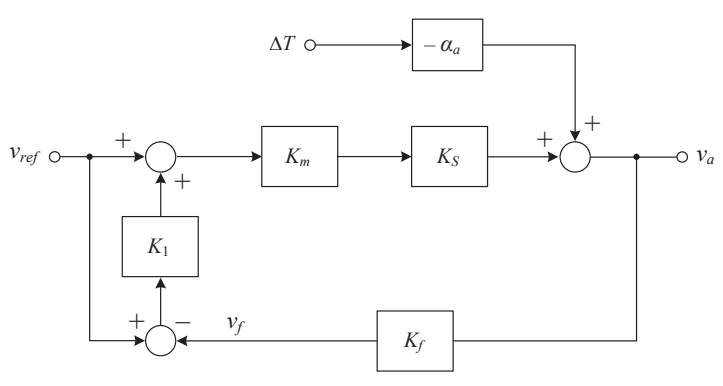

(b)

Fig. 1. (a) Principle of proposed technique and (b) block diagram.

$$
v_{S 2}=K_{L} l_{C}\left(1-\frac{l}{l_{C}}\right)(1-\alpha \Delta T) V_{P} \sin \left(\omega_{e x} t+\phi_{S}\right),
$$

where $K_{L}$ is the sensitivity of the LVDT, $l_{C}$ is the center position of the LVDT, $l$ is the core position deviating from the center position, $\alpha$ is the temperature coefficient, $\Delta T$ is the temperature deviation from the room temperature of $25{ }^{\circ} \mathrm{C}$, and $\phi_{S}$ denotes the phase shift between the primary winding signal and the secondary winding signals. The LVDT output signal $v_{d}$ is the difference between the two secondary winding signals $v_{S 1}$ and $v_{S 2}$ and can be expressed as

$$
v_{d}=v_{S 1}-v_{S 2}=2 K_{L} l(1-\alpha \Delta T) V_{P} \sin \left(\omega_{e x} t+\phi_{S}\right) .
$$

It can be seen that the amplitude of the LVDT signal $v_{d}$ in Eq. (2) is proportional to the core position $l$ and the variation in ambient temperature, $\Delta T$. From Fig. 1(a), the sum voltage $v_{\text {sum }}$ can be stated as

$$
v_{\text {sum }}=-\left(v_{S 1}+v_{S 2}\right)=-K_{S}(1-\alpha \Delta T) V_{P} \sin \left(\omega_{e x} t+\phi_{S}\right),
$$

where $K_{S}=2 K_{L} l_{C}$. Note that the signals $v_{d}$ and $v_{\text {sum }}$ are $180^{\circ}$ out of phase. The signal $v_{\text {sum }}$ in Eq. (3) is directly proportional to the change in ambient temperature without the core displacement $l$. 
To compensate for the temperature effect, the positive-peak amplitude of the signal $v_{\text {sum }}$ is converted to the dc voltage $v_{a}$ and compared with the reference voltage $v_{r e f}$ as shown in Fig. 1(a). The operation of the proposed technique is based on the assumption that the period of the excitation frequency is much less than the change in ambient temperature. The peak-amplitude finder provides the control signal for two SHCs to sample the positive-peak amplitudes of the signals $v_{\text {sum }}$ and $v_{d}$ to the signal $v_{a}$ and the displacement signal $v_{d l}$, respectively. The voltage $v_{a}$ is attenuated by the attenuator $K_{f}$ to provide the signal $v_{f}$ as the feedback signal. From Fig. 1(a), the reference voltage $v_{r e f}$ and the voltage $v_{f}$ are provided for the difference and sum to obtain the signal $v_{m}$ for the voltage-controlled amplifier $A_{m u l}$. For steady-state operation, the voltage $v_{f}$ is forced to equal the reference voltage $v_{r e f}$. The signal $v_{\sin }$ is scaled by the signal $v_{m}$ to provide the excitation signal $v_{e x}$ for the LVDT. The signal $v_{e r r}$ is the difference between the signals $v_{r e f}$ and $v_{f}$, which is processed by the block diagram $K_{1}$, to add with the reference voltage $v_{\text {ref. }}$. The relationship of the signals $v_{m}, v_{r e f}$, and $v_{f}$ can be expressed as

$$
v_{m}=v_{r e f}+K_{1}\left(v_{r e f}-v_{f}\right)
$$

From Fig. 1(a), the signal $v_{\text {sin }}$ is provided with constant amplitude and frequency. The diagram in Fig. 1(a) with the temperature effect can be simplified as shown in Fig. 1(b), where $K_{m}$ is the voltage gain of the voltage-controlled amplifier $A_{m u l}$ and $\alpha_{a}=\alpha K_{S}$. The signal $v_{a}$ against the reference voltage $v_{\text {ref }}$ and the variation in ambient temperature $\Delta T$ can be stated as

$$
v_{a}=\frac{K_{m} K_{S}\left(1+K_{1}\right)}{1+K_{1} K_{f} K_{m} K_{S}} v_{r e f}-\frac{\alpha_{a}}{1+K_{1} K_{f} K_{m} K_{S}} \Delta T
$$

If the gain $K_{f}$ in Eq. (5) is assigned to equal $1 / K_{m} K_{S}$, then the signal $v_{a}$ can be expressed as

$$
v_{a}=K_{m} K_{S} v_{r e f}-\frac{\alpha_{a}}{1+K_{1}} \Delta T
$$

For the proposed technique, the block diagram $K_{1}$ is replaced by the integrating amplifier. Therefore, the signal $v_{e r r}$ of the difference and sum with integral action can be expressed as

$$
v_{e r r}(s)=v_{r e f}(s)-\frac{v_{r e f}(s)-v_{f}(s)}{T_{I} s},
$$

where $T_{I}$ denotes the integral time of the integrating amplifier. Therefore, the voltage $v_{a}$ in Eq. (6) can be rewritten as

$$
v_{a}(s)=K_{m} K_{S} v_{r e f}(s)-\frac{\alpha_{a} T_{I} s}{\left(1+T_{I} s\right)} \Delta T(s) .
$$

The circuit diagram of the proposed technique in Fig. 1(b) is shown in Fig. 2. The operation 


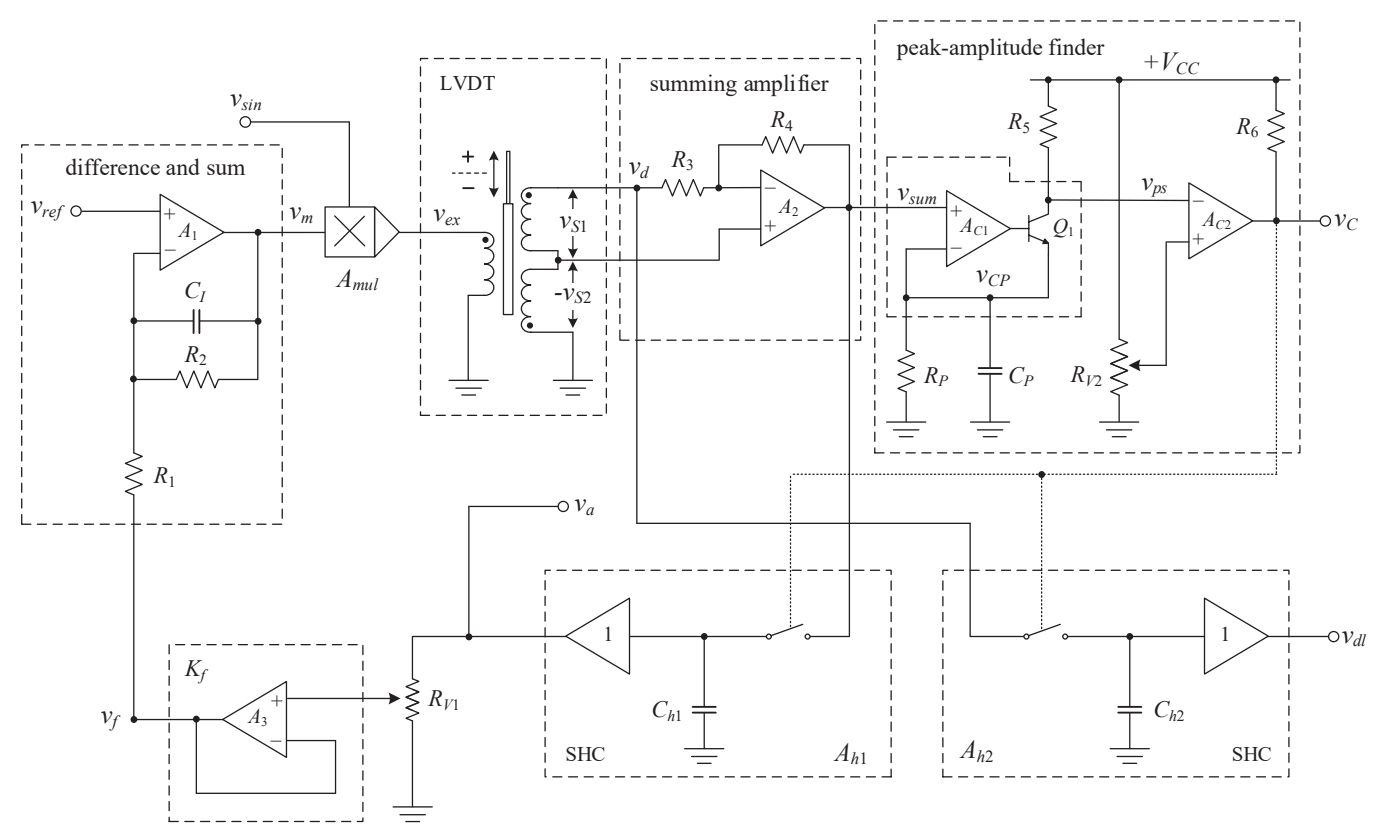

Fig. 2. Circuit of proposed technique.

of the proposed circuit in Fig. 2 can be explained as follows. The LVDT output signal $v_{d}$ and the signal $v_{S 2}$ are summed using the summing amplifier formed by the opamp $A_{2}$ and the resistors $R_{3}$ and $R_{4}$ to provide the signal $v_{\text {sum. }}{ }^{(8)}$ The peak-amplitude position of the signal $v_{\text {sum }}$ is detected by the peak-amplitude finder that consists of the comparators $A_{C 1}$ and $A_{C 2}$, the transistor $Q_{1}$, the capacitance $C_{P}$, the variable resistor $R_{V 2}$, and the resistors $R_{P}, R_{5}$, and $R_{6}$. The peak-amplitude finder generates the control signal $v_{C}$ in the form of narrow pulse that forces the SHCs $A_{h 1}$ and $A_{h 2}$ to sample the peak amplitudes of the signals $v_{\text {sum }}$ and $v_{d}$ to the voltages $v_{a}$ and $v_{d l}$, respectively. The sampled signal $v_{a}$ is attenuated by the variable resistor $R_{V 1}$ and the opamp $A_{3}$ to provide the feedback voltage $v_{f}=K_{f} v_{a}$. At room temperature $\left(25^{\circ} \mathrm{C}\right)$, the feedback signal $v_{f}$ is equal to the reference voltage $v_{\text {ref }}$. The opamp $A_{1}$, the capacitance $C_{I}$, and the resistances $R_{1}$ and $R_{2}$ form the difference and sum with integral action. The resistance $R_{2}$ is provided to prevent the unstable operation of the opamp $A_{1}$ due to its parasitic capacitances. The output voltage $v_{m}$ of the opamp $A_{1}$ can be given by

$$
v_{m}(s)=\frac{\left(R_{1}+R_{2}\right)}{R_{1}} \frac{\left[\frac{R_{1} R_{2}}{\left(R_{1}+R_{2}\right)} C_{I} s+1\right]}{\left(R_{2} C_{I} s+1\right)} v_{r e f}(s)-\frac{R_{2}}{R_{1}\left(R_{2} C_{I} s+1\right)} v_{f}(s) .
$$

Practically, the resistance $R_{2}$ is assigned to be much greater than the resistance $R_{1}$. Thus, Eq. (9) can be approximated for $\omega>0$ as

$$
v_{m}(s)=\frac{\left(R_{1} C_{I} s+1\right)}{R_{1} C_{I} s} v_{r e f}(s)-\frac{1}{R_{1} C_{I} s} v_{f}(s) .
$$


The voltage $v_{m}$ is fed to the voltage-controlled amplifier $A_{m u l}$ to scale the amplitude of the signal $v_{\sin }$. Therefore, the signal $v_{a}$ of the proposed circuit in Fig. 2 can be stated as

$$
v_{a}(s)=K_{m} K_{S} v_{r e f}(s)-\frac{\alpha_{a} R_{1} C_{I} s}{\left(1+R_{1} C_{I} s\right)} \Delta T(s) .
$$

From Eqs. (8) and (11), the integral time $T_{I}$ is equal to $R_{1} C_{I}$. It can be seen that the first term on the right-hand side of Eq. (11) exhibits the sensitivity of the LVDT without interference by the proposed feedback technique. The proportional-plus-integral action occurs only in the second term on the right-hand side of Eq. (11). It can be seen that the effect of the variation in ambient temperature $\Delta T$ is eliminated by the integral action. In addition, the core position $l$ can be extracted from the positive-peak amplitude of the LVDT output signal $v_{d}$ in the form of the displacement signal $v_{d 1}$ by using the SHC $A_{h 2} \cdot{ }^{\left({ }^{8}\right)}$ Therefore, the temperature effect in the displacement signal $v_{d l}$ is also avoided.

\section{Circuit Analysis}

The ideal performance of the proposed circuit can be disturbed by the nonideal characteristic of devices used in the proposed scheme and the sampling rate of the SHC. The circuit analysis of the proposed scheme can be explained as follows. For the peak amplitude finder, the control signal $v_{C}$ can be discontinued by the inappropriate value of the time constant $T_{P}=R_{P} C_{P}$. To prevent the discontinued pulse of the control signal $v_{C}$, the time constant $T_{P}$ is assigned as $\left(T_{e x}-T_{q}\right)$, where $T_{e x}$ and $T_{q}$ denote the period of the excitation frequency and the acquisition time of the SHC, respectively. From Fig. 2, the positive-peak amplitude of the voltage $v_{\text {sum }}$ is equal to $K_{S} V_{P}$. The voltage $v_{C P}$ is fallen to the voltage $K_{S} V_{P} \sin \left[(1 / 2) \pi-\left(2 \pi T_{q} / T_{e x}\right)\right]$ in the period of $\left(T_{e x}-T_{q}\right)$ after the peak-amplitude position of the voltage $v_{\text {sum }}$. Thus, the time constant $T_{P}$ can be stated as

$$
T_{P}=R_{P} C_{P}=\frac{T_{e x}-T_{q}}{\ln \frac{1}{\sin \left(\frac{T_{e x}-4 T_{q}}{2 T_{e x}}\right) \pi}} .
$$

To minimize the ripple voltage of the SHC output, the pulse width of the control signal $v_{C}$ is set to equal the acquisition time $T_{q}$ of the SHC and provided by the time constant $T_{W}=R_{5} C_{P}$. From Fig. 2, the resistor $R_{5}$ is used to convert the collector current $i_{C 1}$ of the transistor $Q_{1}$ to the voltage signal $v_{p s}$. The comparator $A_{C 2}$ and the variable resistor $R_{V 2}$ are provided for shaping the signal $v_{p s}$ to the control signal $v_{C}$. The collector current $i_{C 1}$ of the transistor $Q_{1}$ is allowed to flow through the capacitor $C_{P}$ and the resistor $R_{P}$, which can be expressed as

$$
i_{C 1}=\frac{V_{O P}\left[1-\sin \left(\frac{T_{e x}-4 T_{q}}{2 T_{e x}}\right)\right] C_{P}}{T_{q}},
$$


where $V_{O P}$ denotes the peak amplitude of the voltage signal $v_{\text {sum }}$. The voltage across the resistor $R_{5}$ is equal to $i_{C 1} R_{5}$ or $\left(V_{C C}-V_{O P}\right)$. Therefore, the relationship of the time constants $T_{W}$ and $T_{q}$ can be given by

$$
T_{W}=R_{5} C_{P}=\frac{\left(V_{C C}-V_{O P}\right) T_{q}}{V_{O P}\left[1-\sin \left(\frac{T_{e x}-4 T_{q}}{2 T_{e x}}\right) \pi\right]} .
$$

If $T_{q}=6.5 \mu \mathrm{s}, f_{e x}=5 \mathrm{kHz}, V_{O P}=2.92 \mathrm{~V}, V_{C C}=12 \mathrm{~V}$, and $C_{P}=0.1 \mu \mathrm{F}$, then the resistances $R_{P}$ and $R_{5}$ can be calculated as 92.16 and $9.7 \mathrm{k} \Omega$, respectively. For stability consideration, the transfer function $F_{S H}(s)$ of the SHC can be approximated using the first-order Padé approximation for the sampling time $T_{e x}$ as ${ }^{(17)}$

$$
F_{S H}(s)=\frac{e^{-T_{e x} s}}{\left(1+T_{S H} s\right)}=\frac{\left(-\frac{T_{e x}}{2} s+1\right)}{\left(\frac{T_{e x}}{2} s+1\right)\left(T_{S H} s+1\right)},
$$

where $T_{S H}$ and $T_{e x}$ denote the response time of the SHC and the period of excitation frequency, respectively. Figure 3 shows a block diagram of Fig. 2 including the nonideal characteristics of active devices used in the proposed scheme. From Fig. 3, $T_{s u}$ and $T_{m}$ are time constants of the opamp $A_{2}$ with unity gain and the response time of voltage-controlled amplifier $A_{m u l}$, respectively. The time constant $T_{s u}$ of the opamp $A_{2}$ is equal to $1 / G B P$, where $G B P$ is the gain bandwidth product of the opamp $A_{2}$. Note that the time constants $T_{s u}, T_{m}$, and $T_{S H}$ are much less than the period of the excitation frequency. Therefore, the transfer function of the proposed scheme in Fig. 3 can be approximated as

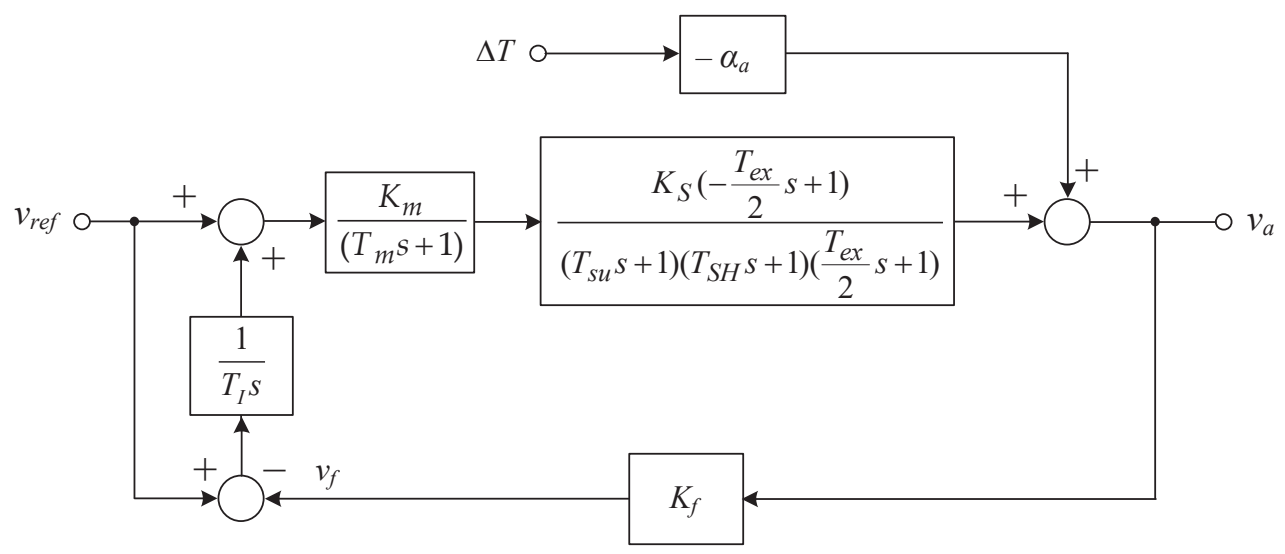

Fig. 3. Block diagram of Fig. 2. 


$$
\begin{aligned}
v_{a}(s)= & \frac{\left[\left(T_{I}-\frac{T_{e x}}{2}\right) s+1\right] K_{m} K_{S}}{\left(T_{I}-\frac{K_{m} K_{S} K_{f} T_{e x}}{2}\right) s+K_{m} K_{S} K_{f}} v_{r e f}(s) \\
& -\frac{\alpha_{a} T_{I} s\left[\left(T_{m}+T_{s u}-\frac{T_{e x}}{2}\right) s+1\right]}{\left(T_{I}-\frac{K_{m} K_{S} K_{f} T_{e x}}{2}\right) s+K_{m} K_{S} K_{f}} \Delta T(s) .
\end{aligned}
$$

Practically, the feedback gain $K_{f}$ is assigned as $1 / K_{m} K_{S}$. Also, the degree of change in ambient temperature is much lower than the excitation frequency. Then, the zeros of the second term on the right-hand side of Eq. (16) satisfy the condition of $\left|\left(T_{m}+T_{s u}-T_{e x} / 2\right) \omega\right|<<1$ and Eq. (16) can be approximately given by

$$
v_{a}(s)=\frac{\left[\left(T_{I}-\frac{T_{e x}}{2}\right) s+1\right] K_{m} K_{S}}{\left(T_{I}-\frac{T_{e x}}{2}\right) s+1} v_{r e f}(s)-\frac{\alpha_{a} T_{I} s}{\left(T_{I}-\frac{T_{e x}}{2}\right) s+1} \Delta T(s) .
$$

From Eq. (17), the condition of $T_{I}>T_{e x} / 2$ is assigned for stability consideration. The steadystate behavior against the signal $v_{r e f}$ for $K_{m}=1$ can be given by

$$
v_{a}=K_{S} v_{r e f}=2 K_{L} l_{C} v_{r e f}
$$

It can be seen that the amplitude of the signal $v_{a}$ is only dependent on the sensitivity of the LVDT. If the change in ambient temperature, $\Delta T$, is considered, then the derivation of the signal $v_{a}$ against the temperature $\Delta T$ can be written as

$$
v_{a}(s)=\frac{\alpha_{a} T_{I} s}{\left(T_{I}-\frac{T_{e x}}{2}\right) s+1} \Delta T(s) .
$$

Practically, the change in ambient temperature, $\Delta T$, is slow against the excitation frequency, and the time constant $T_{I}$ is much larger than the period $T_{e x}$ of the excitation frequency $f_{e x}$ to prevent the phase shift caused by the sampling signal of the SHC. Therefore, Eq. (19) can be approximated as

$$
v_{a}(s)=\frac{\alpha_{a} T_{I} s}{T_{I} s+1} \Delta T(s) .
$$

The integral time $T_{I}$ can be determined as $T_{I} \geq 10 T_{e x}$. From the circuit in Fig. 2, the settling time $T_{S}$ for the sudden change in ambient temperature, $\Delta T$, can be measured from the interval time of the maximum change in the signal $v_{a}$ to decrease to $0.1 \%$ and can be given by

$$
T_{S}=6.908 T_{I} .
$$


Practically, the integral time $T_{I}$ is assigned as $10 T_{e x}$ for the fast response time. Then, the settling time $T_{S}$ is about $13.816 \mathrm{~ms}$.

\section{Experimental Results}

The proposed circuit in Fig. 2 was implemented to confirm the circuit performance. The active devices used in the circuit were commercially available devices such as the opamp LF351 for $A_{1}$ and $A_{2}$, the voltage-controlled amplifier $A_{m u l}$ formed by the analog multiplier AD633, the SHC LF398 for $A_{h 1}$ and $A_{h 2}$, and the comparator LM339 for $A_{C 2}$. The comparator $A_{C 1}$ and the transistor $Q_{1}$ were provided in the same integrated circuit package as LM311. The GBPs of the opamps $A_{1}$ and $A_{2}$ are about $3 \mathrm{MHz}$ and the time constant $T_{s u}$ can be approximated as $53 \mathrm{~ns}$. The LVDT of $\pm 12.5 \mathrm{~mm}$ stroke range and the sensitivity of $69 \mathrm{mV} / \mathrm{mm} / \mathrm{V}$ at $5 \mathrm{kHz}$ were used in this study. ${ }^{(8)}$ The voltage gain $K_{m}$ of the voltage-controlled amplifier $A_{m u l}$ was set to 1. The resistors $R_{3}=R_{4}=30 \mathrm{k} \Omega$ and $R_{V 1}=R_{V 2}=10 \mathrm{k} \Omega$ were assigned. The supply voltages were set to $\pm 12 \mathrm{~V}$. The excitation signal $v_{\sin }$ was assigned as a $5 \mathrm{kHz}$ sinusoidal wave of $2 \mathrm{~V}$ peak-to-peak voltage, where the period $T_{e x}$ of the excitation frequency was calculated as 0.2 ms. The reference voltage $v_{r e f}$ was set to $1 \mathrm{~V}$. The capacitances $C_{h 1}=C_{h 2}=0.01 \mu \mathrm{F}$ were used for the SHCs $A_{h 1}$ and $A_{h 2}$, which caused the acquisition time of about $T_{q}=6.5 \mu \mathrm{s}$. For the peak amplitude finder, the capacitance $C_{P}$ was chosen as $0.1 \mu \mathrm{F}$. Therefore, the resistances $R_{P}$ and $R_{5}$ were determined from Eqs. (12) and (14) as 92.16 and $9.73 \mathrm{k} \Omega$, respectively. From Eq. (21), the integral time $T_{I}$ was assigned to equal $10 T_{e x}$ or $2 \mathrm{~ms}$ for the fast response time and the resistor $R_{1}=20 \mathrm{k} \Omega$ was calculated for the capacitance $C_{I}$ set to $0.1 \mu \mathrm{F}$. The resistance $R_{2}=300 \mathrm{k} \Omega$ was assigned for the condition of $R_{2} \gg R_{1}$ to avoid the unstable operation of the opamp $A_{1}$. The variable resistor $R_{V 1}$ was adjusted for scaling the voltage $v_{f}$ equal to the reference voltage $v_{r e f}$ at room temperature. Figure 4 shows the signal waveforms $v_{e x}$ and $v_{s u m}$ of the proposed scheme in Fig. 2. It also shows the phase shift between the signals $v_{e x}$ and $v_{\text {sum }}$ of about $18^{\circ}$. The output

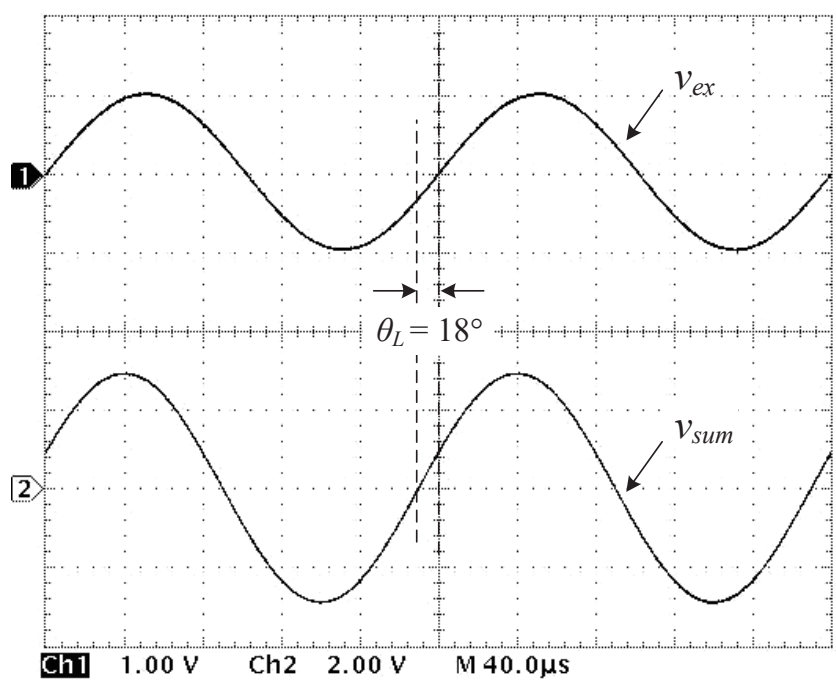

Fig. 4. Measured waveforms of $v_{e x}$ and $v_{\text {sum }}$. 
voltage $v_{a}$ of the SHC $A_{h 1}$ is equal to the peak amplitude of the signal $v_{\text {sum }}$ because the sampling signal $v_{C}$ occurred at the peak-amplitude position of the signal $v_{\text {sum. }}$. To verify the temperature performance, the LVDT was operated under the varying ambient temperature from 20 to 70 ${ }^{\circ} \mathrm{C}$ and the varying core position of the LVDT from -10 to $10 \mathrm{~mm}$. The relative error of the measured results in percentage can be expressed as

$$
\text { relative } \operatorname{error}(\%)=\frac{|\Delta l|}{l} \times 100 \% \text {, }
$$

where $\Delta l$ denotes the error between the measured and actual values. The temperature effect in the core displacement signal $v_{d}$ without temperature compensation is shown in Fig. 5(a). Figure 5(b) shows the measured results of the core displacement signal with the temperature compensation. It can be seen that the proposed technique can reduce the maximum relative error from 6.52 to $0.098 \%$. Figure 6 shows the drift of the offset voltage of the output signal $v_{d l}$

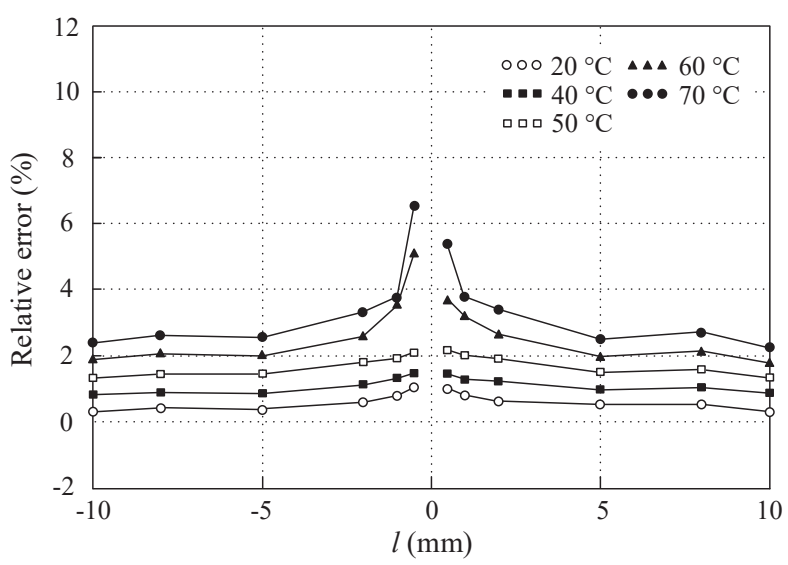

(a)

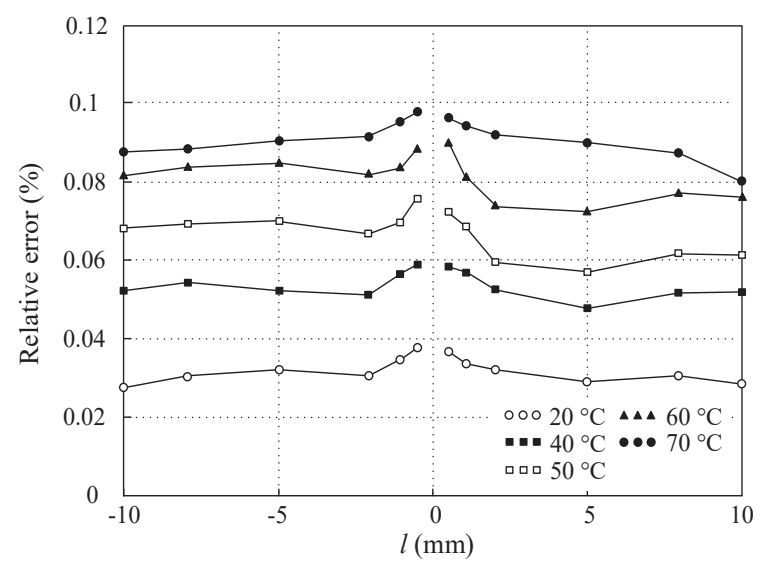

(b)

Fig. 5. Measured results. (a) Without temperature compensation and (b) proposed technique.

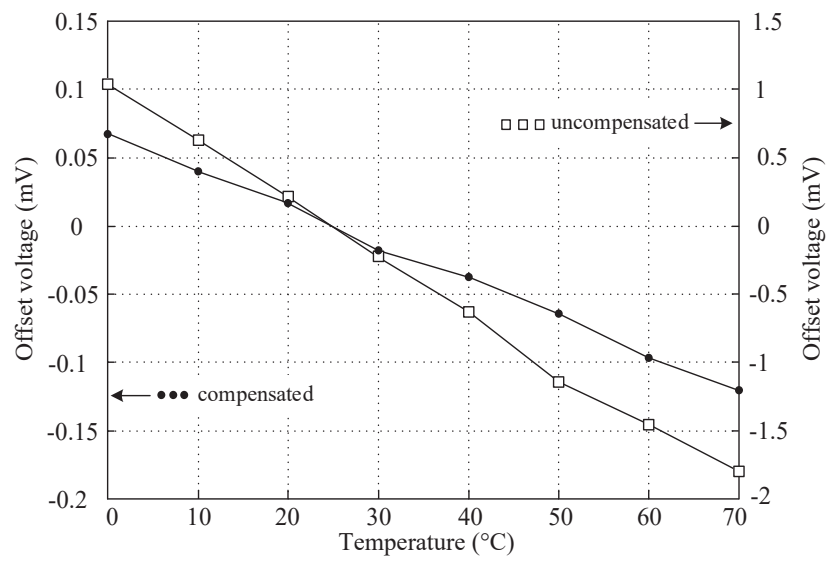

Fig. 6. Offset voltage against variation in ambient temperature. 
against the variation in ambient temperature from 0 to $70{ }^{\circ} \mathrm{C}$ at the core position of $0 \mathrm{~mm}$. From Fig. 6 , the maximum drift of the offset voltage of about $-0.12 \mathrm{mV}$ is observed.

\section{Conclusions}

A technique to reduce the error of the LVDT signal caused by the variation in ambient temperature has been proposed. The effect of the change in ambient temperature was discussed in detail. The proposed technique required the integral action to reduce the error caused by the change in ambient temperature. The proposed circuit was implemented using commercially available devices. The performance characteristics of the proposed circuit were discussed and confirmed by experimental implementation. It is evident that the proposed scheme provides adequate performance for the applications of the LVDT in an environment with a highly fluctuating temperature such as a steam control valve, a power turbine, a nuclear reactor, a flight control actuation system, a variable area nozzle for aircraft, and an automotive active suspension system.

\section{Acknowledgments}

This work was supported by the King Mongkut's Institute of Technology Ladkrabang (KMITL), Thailand, under Grant No. KREF115701.

\section{References}

1 R. Pallas-Areny and J. G. Webster: Sensor and Signal Condition (Wiley, New York, 2001) p. 229.

2 S. Cetinkunt: Mechatronics (Wiley, New York, 2006) p. 227.

3 S. Lee and W. Kim: IEEE Trans. Control Syst. Technol. 18 (2010) 859.

4 M. Felix, A. Lizarraga, A. Islas, and A. Gonzales: 36th Conf. IEEE Industrial Electronics Society (IECON, 2010) 1769-1772.

5 G. Chen, B. Zhang, P. Liu, and H. Ding: IEEE Sensors J. 15 (2015) 2248.

6 J. Lee, K. C. Lee, S. Cho, and S. H. Sim: Sensors 17 (2017) 2317.

7 W. Petchmaneelumka, K. Songsuwankit, A. Rerkratn, and V. Riewruja: 3rd Int. Conf. Control, Automation and Robotics (ICCAR 2017) 758-761.

8 W. Petchmaneelumka, K. Songsuwankit, and V. Riewruja: Int. Rev. Electr. Eng. 11 (2016) 340.

9 W. Petchmaneelumka, K. Songsuwankit, and V. Riewruja: 9th Int. Conf. Computer and Automation Engineering (ICCAE 2017) 193-197.

10 A. Drumea, A. Vasile, M. Comes, and M. Blejan: 1st Electronic Systemintegration Technology Conf. (ESTC 2006) 629-634.

11 R. Casanella, O. Casas, and R. Pallas-Areny: Meas. Sci. Tech. 16 (2005) 1637.

12 C. S. Koukourlis, V. K. Trigonidis, and J. N. Sahalos: IEEE Trans. Instrum. Meas. 42 (1993) 926.

13 D. A. Johns and K. Martin: Analog Integrated Circuit Design (Wiley, New York, 1997) p. 389.

14 K. Ara: IEEE Trans. Instrum. Meas. IM-21 (1972) 249.

15 S. C. Saxena and S. B. L. Seksena: IEEE Trans. Instrum. Meas. 38 (1989) 748.

16 T. S. Smith: U.S. Patent US 5087866 A (1991).

17 F. Golnaraghi and B. C. Kuo: Automatic Control System (Wiley, New York, 2010) p. 206. 